\title{
PEDAGOGÍAS CREATIVAS Y TRABAJO POR PROYECTOS EN LA FORMACIÓN MUSICAL EN LOS GRADOS DE EDUCACIÓN. EL CASO DE LA FACULTAD DE EDUCACIÓN DE PALENCIA
}

Recepción: 22/09/2019 | Revisión: 21/10/2019 | Aceptación: 06/12/2019

\author{
Alicia PEÑALBA \\ Universidad de Valladolid \\ alicia.penalba@uva.es
}

\author{
Yurima BLANCO GARCÍA \\ Universidad de Valladolid \\ yurima.blanco.garcia@uva.es
}

\begin{abstract}
Resumen: El presente artículo expone diversas experiencias llevadas a cabo en la formación musical universitaria, en los Grados de Educación Infantil, Primaria y Social de la Facultad de Educación de Palencia, Universidad de Valladolid. Tomando como referencia las pedagogías creativas y el trabajo por proyectos, se muestran propuestas desarrolladas por el alumnado en la clase de Música bajo un enfoque globalizador. Estos proyectos tratan de hacer frente a tres importantes retos que caracterizan la educación en el siglo XXI: el componente creativo de la educación, la responsabilidad social, y el uso de las Tecnologías de la Información y las Comunicaciones (TICs) de forma crítica y responsable. Las ideas pedagógico-musicales desarrolladas por Paynter, Delalande y Alcázar, y los proyectos de transformación social a través de la música -como el conocido "Sistema" de orquestas en Venezuela- se convierten en puntos de partida para generar nuevas experiencias pedagógicas en la formación inicial del profesorado y para dar respuesta a las demandas de la educación musical en nuestra sociedad.
\end{abstract}

Palabras clave: formación de educadores; Aprendizaje Basado en Proyectos (ABP) y música; creatividad; responsabilidad social; TICs.

\section{CREATIVE PEDAGOGIES AND PROJECTWORK INTHE TRAINING OF UNIVERSITYTEACHERS. THE CASE OF THE FACULTY OF EDUCATION OF PALENCIA}

\begin{abstract}
This article presents various experiences carried out in Higher Education training of Pre-school, Primary and Social Educators in the Faculty of Education of Palencia (University of Valladolid, Spain). Taking as a reference the Creative Pedagogies and the Project Based Learning, the proposals developed by the students in the Music class under a globalizing approach are shown. These projects try to face three important challenges that characterize education in the 21st century: the creative component of education, social responsibility, and the use of Information and Communication Technologies in a critical and responsible way. The pedagogicalmusical ideas developed by Paynter, Delalande and Alcázar, and the projects of social transformation through music-such as the well-known "Sistema de orquestas" in Venezuela- become starting points to generate new pedagogical experiences in the initial training of educators to respond to the demands of musical education in our society.
\end{abstract}

Keywords: teacher training; project-based learning and music; creativity; social responsibility; ICT tools.
PEDAGOGIES CREATIVES ITREBALL PER PROJECTES EN LA FORMACIÓ MUSICAL EN ELS GRAUS D'EDUCACIÓ. EL CAS DE LA FACULTAT D'EDUCACIÓ DE PALĖNCIA

Resum: Aquest article exposa diverses experiències dutes a terme en la formació musical universitària en els Graus d'Educació Infantil, Primària i Social de la Facultat d'Educació de Palència, Universitat de Valladolid. Partint del referent de les pedagogies creatives $i$ el treball per projectes, es mostren propostes desenvolupades pels estudiants a la classe de Música seguint un enfocament globalitzador. Aquests projectes s'enfronten a tres reptes que caracteritzen l'educació en el segle XXI: el component creatiu de l'educació, la responsabilitat social, $i$ l'ús de les Tecnologies de la Informació $i$ Comunicació (TICs) de forma crítica i responsable. Les idees pedagògicomusicals desenvolupades per Paynter, Delalande i Alcázar, i els projectes de transformació social a través de la música-com el conegut "Sistema" d'orquestres a Veneçuela- es converteixen en punts de partida per generar noves experiències pedagògiques en la formació inicial del professorat $i$ per respondre a les demandes de l'educació musical a la nostra societat.

Paraules clau: formació d’educadors; Aprenentatge Basat en Projectes (ABP) $i$ música; creativitat; responsabilitat social; TICs. 


\section{Introducción}

La educación musical actual está atravesando un importante declive (Aróstegui, 2003, p. 2). Por un lado, las leyes educativas están haciéndola desaparecer casi por completo del currículo obligatorio y, por otro, la escuela está sufriendo importantes cambios derivados de una significativa transformación social. Los sistemas educativos están afrontando "profundos cambios globales, las transformaciones socioeconómicas y científico-tecnológicas generan nuevas condiciones, discursos y procesos, que desafían las estructuras tradicionales" (García Pedraza, 2015, p. 136).

A pesar de esta menor presencia de la música en la escuela, esta ocupa un importante lugar en la sociedad, en el ocio, y en las vidas cotidianas del alumnado de la educación obligatoria. Son numerosos los estudios que avalan su importancia para el desarrollo cognitivo, emocional, interpersonal, identitario y social (Peñalba, 2017). Estos estudios muestran en su mayoría que la música proporciona efectos de transferencia a diversas áreas como la lengua, el pensamiento matemático, la percepción visoespacial, entre otros (Hallam, 2010). Si esto es así ¿por qué la educación musical no tiene el impacto esperado en la escuela (Cremades-Andreu, 2019) y ¿qué debe hacerse para satisfacer los retos de la educación obligatoria en la actualidad? ${ }^{1}$

Sin duda, las respuestas a esta pregunta entrañan gran complejidad. Algunas de ellas se abordan en los resultados del proyecto IMPACTMUS, que se ha detenido en estudiar el impacto que la educación musical ha tenido en la sociedad y la economía del conocimiento ${ }^{2}$. De los resultados, se infiere que algunos de los retos para la educación musical del siglo XXI deben tener en cuenta una formación musical a través de aprendizajes significativos, más creativa, socialmente comprometida y adaptada a los nuevos tiempos y lenguajes (Carrillo y Pérez-Moreno, 2019).

Otro de los resultados principales de ese proyecto muestra que tener buenos docentes de música es la clave para que la educación musical tenga impacto en el alumnado (Serrano y Peñalba, 2019). Por esa razón, el presente artículo se centra en la formación musical de educadores y de cómo en la Facultad de Educación de Palencia abordamos dicha formación -de profesorado generalista de Educación Infantil, Educación Primaria y Educación Social- tratando de dar soluciones a los importantes retos con los que se encuentra la escuela en la actualidad. Estas soluciones parten de un trabajo por proyectos con el alumnado. Los proyectos que emprendemos en nuestra facultad van orientados hacia el desarrollo de competencias creativas, cívicas y de uso crítico de las tecnologías de la información y la comunicación.

\section{El trabajo por proyectos como vertebrador de aprendizajes}

En la educación obligatoria actual, nos hemos visto sometidos a una compartimentación de los saberes, a pesar de que las personas aprendemos de forma global mucho más que de manera parcial y especializada. Ventura (1996) señala que las bases de la noción globalizadora se centran en la manipulación del medio como forma de generar la interiorización conceptual. En palabras de Suárez (2000):

1 Actualmente las autoras forman parte del Proyecto de investigación I+D EDU2017-84782-P sobre Formación del Profesorado y Música en la Sociedad y en la Economía del Conocimiento, financiado por el Ministerio de Economía y Competitividad del Gobierno de España.

2 Referencia EDU2014-58066-P (2015-2018), financiado por el Ministerio de Economía y Competitividad de España. 
Una de las ideas básicas de la globalización y la transversalidad en la práctica educativa tiene que ver con la oposición de esta concepción epistemológica y curricular a la tradicional, basada en la linealidad del conocimiento, el carácter acumulativo de la ciencia y la especialización del saber, que ha permanecido en la escuela y se pretende erradicar, al contradecirse con la cultura de la época (p.49).

El Aprendizaje basado en proyectos (ABP), metodología descrita por Kilpatrick (1918) se basa en la propuesta de un proyecto de trabajo que tiene una finalidad real, que orienta los procedimientos de trabajo y les confiere una motivación. La técnica gira en torno al diseño y elaboración de un objeto (por ejemplo, un terrario, un huerto escolar, una maqueta o un periódico) o bien la realización de un montaje (una representación de teatro, un audiovisual, una danza, una pieza musical), siendo fundamental seguir sistemáticamente las fases de intención, preparación, ejecución y evaluación (Pareja, 2011). El ABP aplicado en los cursos -referido a la Educación Superior en este caso-, proporciona una experiencia de aprendizaje que involucra al estudiante en un proyecto complejo y significativo, mediante el cual desarrolla integralmente sus capacidades, habilidades, actitudes y valores (Maldonado-Pérez, 2008, p. 160).

Emplear el ABP como estrategia didáctica se considera relevante en la experiencia educativa, al considerar que: (a) la metodología de proyectos es una estrategia para el aprendizaje que permite el logro de aprendizajes significativos, porque surgen de actividades relevantes para los estudiantes, y contemplan muchas veces objetivos y contenidos que van más allá que los curriculares. (b) Permite la integración de asignaturas, reforzando la visión de conjunto de los saberes humanos. (c) Permite organizar actividades en torno a un fin común, definido por los intereses de los estudiantes y con el compromiso adquirido por ellos. (d) Fomenta la creatividad, la responsabilidad individual, el trabajo colaborativo y la capacidad crítica, entre otros (Maldonado-Pérez, 2008).

Esta forma de trabajo a través de proyectos colaborativos se muestra eficaz con respecto a la motivación del alumnado, el sentido de responsabilidad, la autonomía para el aprendizaje, la integración de saberes, el fomento de las capacidades de trabajo en equipo, el acercamiento a la realidad profesional y la capacidad de evaluar los propios logros. Además, supone un modelo que los propios maestros o educadores sociales pueden implementar con su alumnado en el futuro.

Majó (2001, p. 17) propone una serie de fases que todo proyecto interdisciplinario debería tener y que se respetan en las propuestas de proyectos que se presentarán más adelante:

Fase 1. La elección del tema, que supone: qué se sabe de él, qué se quiere aprender, qué se necesita para aprender, etc. Esta fase es fundamental en la motivación y negociación colectiva. Sólo si el tema parte de los intereses del alumnado supondrá un reto para querer saber. Fase 2. La planificación del trabajo, la organización y temporalización; Fase 3. La elaboración de proyecto propiamente dicho; Fase 4. La evaluación.

\subsection{Pedagogías creativas y proyectos de trabajo}

De acuerdo con Gustems (2013), la música debería aportar una mayor presencia, de forma sistemática e interdisciplinaria, al campo de investigaciones en creatividad. En el ámbito de la educación, ser creativos se considera una competencia transversal y sugiere el desarrollo de 
actividades innovadoras, originales e interesantes que formulen nuevas alternativas en el presente.

Desde el punto de vista de la educación musical, los llamados Pedagogos Creativos tienen en común dos aspectos fundamentales: la consideración del alumno como un creador o compositor; y la integración de competencias a través del desarrollo de proyectos de trabajo, como reflejan las propuestas de Schafer (1994), Paynter (1999), Dennis (Dennis y Schultis, 1991) o Delalande (Blanco-García, 2018). Los proyectos utilizan por lo general repertorios de su contemporaneidad, desde las músicas ligadas a paisajes sonoros (Schafer, 1994) hasta músicas concretas (Delalande, 2004) que son consideradas como más cercanas a las posibilidades creativas de los niños, o en este caso, el alumnado universitario no especialista. Queremos describir aquí algunas de las propuestas de estos pedagogos porque sentarán las bases de diversos proyectos de trabajo implementados en las aulas.

\section{- Los proyectos de Paynter}

Paynter (1999) dedica su libro Sonido y Estructura a proporcionar herramientas para la creación musical. Presenta dieciséis proyectos, cada uno de ellos basado en una propuesta diferente para la creación. Algunos de ellos son los siguientes: Sonidos a partir del silencio es un proyecto en el que se busca que los alumnos experimenten el sonido desde la escucha atenta y desde la producción de sonidos para recrear "ambientes"; Sonidos encontrados trabaja con sonidos producidos por objetos cotidianos; Los dedos son unos grandes inspiradores se centra en cómo la gestualidad puede ser un elemento inspirador de la creación (donde nos llevan los gestos al tocar); Desarrollos necesarios parte de una idea musical que se explota al máximo; Oídos nuevos aborda el estudio de la interpretación de instrumentos tradicionales de forma no convencional; Una estructura clásica utiliza formas tradicionales y clásicas, como la forma sonata, pero con contendidos diversos y lenguajes contemporáneos.

\section{- Los proyectos de Schafer}

Las propuestas de Schafer promueven la sensibilidad y creatividad de los seres humanos respecto al mundo sonoro que les rodea. Sus ideas pedagógico-musicales tienen como núcleo común diseñar una ecología acústica global, percibir "el paisaje sonoro del mundo como una composición musical" (Schafer, 2013, p. 283). El libro Hacia una educación sonora. 100 ejercicios de audición y producción sonora formula diversos proyectos para desarrollar en el aula: entre ellos destacan: Anote todos los sonidos que escucha, que busca escuchar y reflexionar sobre las fuentes emisoras, la intensidad del sonido, sus peculiaridades, etc; Los sonidos móviles que propone emitir sonidos y moverse por la sala y escuchar el sonido en movimiento; Caminata auditiva (listening walk) que aboga por la consciencia de los sonidos del entorno; Diario de sonidos, que se centra en aquellos sonidos que son significativos, especiales y nos acompañan en nuestra cotidianidad; La hoja de papel es un instrumento musical, que explora las posibilidades sonoras de este material.

\section{- Los proyectos de Antonio Alcázar}

Alcázar (2013, pp. 20-29), siguiendo algunos de los principios de la Pedagogía de la Creación 
Musical propuesta por Delalande, Vidal, y Reibel (2001) propone seis ideas para seis proyectos que fomentan la creatividad en el aula: Interpretar-recrear a partir de una canción infantil para crear nuevas piezas musicales inspiradas en estas; Componer a partir de un texto, empleando la voz y/o cuerpos sonoros; Escuchar-analizar e improvisar-recrear a partir de varios paisajes sonoros; Escuchar-analizar a partir de una pieza musical empleando el movimiento corporal y la grafía no convencional o Componer a partir de una imagen.

Inspirados en estas propuestas creativas, que son presentadas y recreadas en el aula, el alumnado de los grados de Educación Infantil, Primaria y Social diseña sus propios proyectos.

\subsection{Proyectos de música para la transformación social}

En una sociedad multicultural y cambiante como en la que vivimos es preciso repensar el fin último de la educación. La tendencia escolar hacia la excelencia en materias aisladas de su aplicabilidad práctica en la vida cotidiana, la competitividad y el trabajo individualista plantea un importante problema de base sobre ese propósito. Si la educación tiene como misión formar ciudadanos críticos y emocionalmente competentes que sepan vivir en sociedad (Conejo, 2012) la música en este sentido tiene un poder innegable.

Diversos proyectos utilizan la música como elemento de transformación social por este gran poder de cambio colectivo. Algunos de los ejemplos más significativos son la proliferación de orquestas basadas en la filosofía de "El Sistema" de orquestas de Venezuela o en la transformación del "bairro do Candeal" en Salvador de Bahía, en Brasil.

"El Sistema" de Orquestas de Venezuela, promovido en 1975 por José Antonio Abreu, es una iniciativa que surge para promover la práctica musical colectiva a través de orquestas sinfónicas y coros para ayudar a niños y jóvenes en riesgo de exclusión social. Majno (2012) especifica que se basa en los siguientes principios metodológicos:

- Accesibilidad (permite que cualquier niño o joven pueda participar de esta experiencia independientemente de su situación personal, social o económica).

- Regularidad e intensidad de entrenamiento (es fundamental para proporcionar la experiencia como alternativa a ocupaciones peligrosas).

- Clases colectivas (se prima el trabajo en equipo, ya que logra desarrollar valores de respeto, equidad, compartir, cohesión, trabajo en equipo y cooperación).

- Búsqueda de la calidad estética (no solo es tocar, sino tocar bien, tocar lo mejor posible).

Hay numerosos proyectos inspirados en El Sistema diseminados en todo el mundo. En España algunas de ellas son Acción social por la Música (Madrid)³, la orquesta "In Crescendo" (Valladolid) ${ }^{4}$, la Fundación Baremboim-Said (Andalucía) ${ }^{5}$ y la Fundación Música Creativa (Madrid) ${ }^{6}$.

3 Realiza actividades con personas en riesgo de exclusión social por situación de pobreza, marginación o discapacidad: http:// accionsocialporlamusica.es/

4 La Orquesta Sinfónica de Castilla León colabora en este proyecto en el que desde hace seis años se ha formado una orquesta y coro con niños de etnia gitana y extranjeros en un colegio público de la capital: http://www.centroculturalmigueldelibes.com/areas/areasocial/proyecto-orquestal-y-coral-infantil-in-crescendo/

5 http://www.barenboim-said.org/es/inicio/index.html

$6 \mathrm{http}: / /$ www.fundacionmusicacreativa.com/ 
Por su parte, otra de las iniciativas punteras en cuanto a la transformación social a través de la música es la iniciativa del "bairro do Candeal", que surge de la mano del músico Carlihnos Brown, que se crió en esta favela y que pudo comprobar cómo la música le permitió salir de la situación de marginación en la que se encontraba. Lauro y Repoll (2011) indican que:

Carlinhos tomó la decisión de trabajar por los niños y jóvenes del barrio, destinando parte del dinero que había ganado en la gira, para acercarlos al mundo de la música como una salida de los problemas relacionados con la delincuencia y la droga existentes en el barrio. En el año 1994 nace la Escuela de Música "Pracatum" y posteriormente el grupo de percusionistas “Timbalada" en el año 1997. La propuesta pedagógico-cultural que sostiene el proyecto de Carlinhos, se basa en los preceptos de la educación popular al partir de los intereses y necesidades de la comunidad. De este modo, se plantean talleres de formación musical, formación política y, a su vez, se promueve la participación en la planificación y mejoramiento de las condiciones materiales y simbólicas del barrio (p. 2).

Otras propuestas trabajan con músicas populares urbanas, como las agrupaciones "Motxila 21"7, con personas con Síndrome de Down, o "Xerock", una banda de rock de personas con discapacidad intelectual. La danza es otro de los lenguajes que se han trabajado para favorecer el arte para todos y la inclusión, como en el Psicoballet de Maite León (Martín, 1998), o el grupo "Eudanza" del colegio O'Pelouro", formado con personas con discapacidad y liderado por Laura Llauder.

Desde el punto de vista de la labor llevada a cabo con alumnado del grado de Educación Social fundamentalmente, pero también de Educación Infantil y Primaria, es fundamental que en los diseños de los proyectos el alumnado tenga en cuenta la capacidad de la música de transformar a la sociedad e implementen buenas prácticas que fomenten la inclusión y el cambio a través de la música como agente catalizador de cambios sociales y a tener una visión de la educación musical que vaya más allá del trabajo exclusivo de aula. Además, en este tipo de proyecto se involucra toda una comunidad y se trabaja de forma interdisciplinar, y tener esa conciencia y esa visión resulta importante para comprender la dimensión y complejidad del fenómeno.

\subsection{Las tecnologías de la información y la comunicación como herramienta para el desarrollo de proyectos}

En la sociedad de la información las TICs son consideradas una herramienta indispensable para la transformación de los modelos educativos y sociales, como se advierte en las políticas adoptadas por el Espacio Europeo de Educación Superior (Robertson y Dale, 2008) Es difícil imaginar nuestro presente sin el uso de la tecnología digital e Internet, inmersos en un entorno hipermediático que impacta y transgrede los procesos de enseñanza-aprendizaje. En la formación universitaria el manejo de las TICs se considera una competencia básica, estrechamente relacionada con las denominadas "competencias clave": "usar herramientas de manera de manera interactiva", "actuar de forma autónoma" e "interactuar en grupos heterogéneos" (Organización para la Cooperación y el desarrollo, 2004).

7 http://motxila21.blogspot.com.es/

8 http://www.xerockme.blogspot.com.es/

$9 \mathrm{http}: / /$ www.opelouro.org/ 
Diversos autores subrayan las potencialidades y recursos que brindan las TICs en el terreno de la educación musical (Delalande, 2004; Giráldez, 2010; Savage, 2007; Serrano, 2017). Por una parte, Savage (2007) evidencia cómo los medios tecnológicos capacitan al alumnado para manipular el material sonoro y señalan un punto de partida que favorece los procesos creativos y performativos de la música. Por otro lado, se cuestiona el enfoque dominante de la "competencia musical tecnológica" en el currículum, su falta de neutralidad y el propósito de plegar al "ser musical" ante los nuevos paradigmas de las industrias creativas (Mansfield, 2009, p. 179). De acuerdo con Serrano (2017), las buenas prácticas y el aprovechamiento de las TICs en la formación musical obligatoria debe ser motivo de reflexión para docentes e instituciones, con el propósito de "conjugar la interacción entre tecnología, contenido curricular y pedagogía" (p. 155).

Uno de los proyectos que potencia la creación sonora a través del uso de la tecnología digital lo llevan a cabo Murillo-Ribes, Riaño-Galán, y Berbel-Gómez (2018, pp. 133-135) en la formación universitaria. Tomando como herramienta el programa soundcoo ${ }^{10}$-software diseñado en la Universidad Politécnica de Valencia- explotan sus posibilidades creativas como parte de un proyecto colaborativo y centrado en el alumnado, ya sea con formación musical previa o no. De esta experiencia se desprenden cuatro fases fundamentales: 1) presentación técnica de la herramienta soundcool -el funcionamiento a través de módulos, la descarga de sonidos o la grabación de ejecuciones propias-; 2) primeras pruebas del programa -exploración de los controles básicos del módulo (velocidad, volumen, play, pause, loop, speed) y sus efectos sonoros, la toma de decisiones y la creación de una pieza a partir de ciertas pautas (en cuanto a estructura, timbre e intensidad)-; 3) organización de equipos y desarrollo de una experiencia interuniversitaria - la formación de grupos mixtos de dos universidades para el diseño y creación de una obra sonora y su interpretación en concierto; por último, 4) valoración del proyecto -se aplicó un cuestionario para conocer las percepciones del alumnado sobre su experiencia con el soundcool-.

Este tipo de experiencia compositiva tiene como soporte principal el dispositivo móvil, sin duda, "el medio que más está cambiando los hábitos de accesos a la música y los medios en todo el mundo" (Yúdice, 2017, p. 109). Se puede contribuir, entonces, a una práctica cultural creativa que convierta el smartphone en una herramienta propicia para la exploración de sonidos digitales o creados in situ, la manipulación del sonido desde su propio núcleo (improvisar sobre las cualidades de timbre, dinámica, intensidad y altura), el registro del entorno acústico, la creación de paisajes sonoros o geofonías (Alcázar, 2013; Schafer, 1994), de collages, de nuevos diseños instrumentales o rejuegos sonoros con un sentido "artístico".

10 Disponible para su descarga gratuita en la web: http://soundcool.org/ 


\section{Proyectos musicales en la formación de maestros en la Facultad de Educación de Palencia de la Universidad de Valladolid}

En la Facultad de Educación de Palencia se forman estudiantes de los Grados de Educación Infantil, Educación Primaria y Educación Social.

El plan de estudios de estas titulaciones comprende asignaturas del área de música a partir del segundo curso, algunas de ellas compartidas con las áreas de expresión plástica y corporal. El profesorado de música también participa en la tutorización de prácticas docentes y la dirección de Trabajos de Fin de Grado, como se describe a continuación (Tabla 1):

\begin{tabular}{|c|c|c|c|}
\hline Curso & Educación Infantil (Plan 399) & Educación Primaria (Plan 405) & Educación Social (403) \\
\hline $2^{\circ}$ & & $\begin{array}{l}\text { Fundamentos y estrategias } \\
\text { didícticas de la educación musical. } \\
\text { (Obligatoria. } 6 \text { ECTS) }\end{array}$ & $\begin{array}{l}\text { Tecnicas de dinamización } \\
\text { social (Obligatoria. } 6 \\
\text { ECTS) }\end{array}$ \\
\hline $3^{\circ}$ & $\begin{array}{l}\text { Fundamentos y propuestas didácticas } \\
\text { en la expresión musical (Obligatoria. } \\
6 \text { ECTS.) }\end{array}$ & $\begin{array}{l}\text { Creación artistica y cultura visual y } \\
\text { musical (Qbligateria. } 4 \text { ECTS) }\end{array}$ & $\begin{array}{l}\text { Animación Sociocultural } \\
\text { (Obligatoria. } 6 \text { ECTS) }\end{array}$ \\
\hline $4^{\circ}$ & $\begin{array}{l}\text { - Expresión y comunicación a través } \\
\text { de la música. (Optativa. } 6 \text { ECTS) } \\
\text {-Análisis de prácticas y diseño de } \\
\text { proyectos educativos de las areas de } \\
\text { expresión. (Optativa. } 6 \text { ECTS) } \\
\text {-Recursos didacticos de las areas de } \\
\text { expresión en Educación Infantil } \\
\text { (Optativa. } 6 \text { ECTS.) } \\
\text { - Prácticum (24 ECTS) } \\
\text {-Trabajo Final de Grado (6ECTS) }\end{array}$ & $\begin{array}{l}\text {-Prácticum (24 ECTS) } \\
\text {-Trabajo Final de Grado (6 ECTS) }\end{array}$ & $\begin{array}{l}\text {-Trabajo Final de Grado ( } 6 \\
\text { ECTS) }\end{array}$ \\
\hline
\end{tabular}

Tabla 1. Asignaturas de Música que se imparten en los Grados de Educación Infantil, Primaria y Social. Fuente: propia.

En este artículo se analizan diversas prácticas educativas llevadas a cabo en las asignaturas mencionadas previamente. Desde el punto de vista metodológico, se realiza una selección de las asignaturas de música ofertadas en los tres planes de estudio mencionados que cumplen la condición de utilizar el aprendizaje basado en proyectos. Para la presentación de los datos, se establecen códigos que se corresponden con los diversos medios, técnicas e instrumentos utilizados (Ibarra y Rodríguez, 2010) que se mostrarán en el apartado siguiente a medida que se expongan los proyectos. Se utilizan como medios: informes escritos (M1), memorias de aula (M2), documentos para el estudiante (diapositivas, guías de lectura) (M3), actividades de aula propuestas por el profesor (M4) y proyectos de trabajos elaborados por los estudiantes (M5). Se utiliza la observación sistemática y el análisis de documentos como técnicas. Los datos se analizan con diversos instrumentos de evaluación creados para tal fin como listas de control (E1), escalas de valoración (E2) y rúbricas (E3) con autoevaluaciones y heteroevaluaciones.

Las asignaturas analizadas tienen en común la perspectiva desde las pedagogías creativas de la educación musical, el uso crítico de las TICs y el diseño de proyectos orientados a la transformación social. A continuación, se describirán a grandes rasgos algunos de los proyectos llevados a cabo en estas titulaciones. 


\subsection{Proyectos creativos para la educación infantil}

Los proyectos de las asignaturas "Fundamentos y Propuestas Didácticas en la Expresión Musical" y "Expresión y Comunicación a través de la música", consisten en el diseño, desarrollo e implementación de un proyecto de trabajo para niños de Educación Infantil sobre un tema que se utiliza como hilo conductor que integre el resto de las áreas de conocimiento. Son proyectos creativos en los que el alumnado debe componer, inventar o adaptar a partir de las ideas de Paynter, Alcázar o Delalande, en ocasiones utilizando las TICs. Todos los proyectos deben contar con adaptaciones curriculares para niños con necesidades educativas especiales y han de ser respetuosos con la igualdad de género, no violencia, trabajo emocional y conciencia crítica.

- Fase 1. La elección del tema. El alumnado es libre para elegir el tema que quiere trabajar. En ocasiones se inspiran de experiencias de prácticas o de manuales donde surgen temas adaptados a niños de entre 3-6 años como: el agua, los medios de transporte, viajes por el mundo, el huerto, los alimentos, el cuerpo humano, mi ciudad, el otoño, entre otros (M3).

- Fase 2. La planificación del trabajo viene condicionada por la organización docente. Como de lo que se trata es de que integren los conocimientos nuevos que van adquiriendo primero diseñan actividades sobre los diversos módulos de trabajo: cuerpos sonoros, voz, cuerpo y escucha. Primero se trabaja la práctica y la teoría se va presentando progresivamente para dar sentido a toda la práctica (M3, M4).

- Fase 3. La puesta en práctica. Se realiza un role-playing en el aula con sus compañeros (M5). En paralelo y a lo largo del proceso van realizando lecturas para poder fundamentar teóricamente el proyecto (M3). El último paso es realizar un informe final que incluya todo el proyecto (M1) y que incorpore lo aprendido a través de las evaluaciones parciales de puesta en práctica de las actividades y la evaluación final propuesta. Dicho informe es revisado y devuelto con correcciones por la profesora y, tras ser modificado se presenta oralmente en el aula utilizando fotografías, pequeñas grabaciones de vídeo, etc. (M2). Una de las actividades se realiza en un encuentro con niños en edad 3-6 de centros educativos de la zona (M5).

- Fase 4. Evaluación. No se realiza al final, como se ha mencionado, sino que se hace una evaluación procesual y otra final. Se evalúan las actividades que presentan los alumnos durante cuatro sesiones prácticas a lo largo del curso y cómo integran los comentarios, observaciones de otros compañeros, de la profesora y sus propias observaciones en la redacción del trabajo final a través de una autoevaluación. La profesora da también un feedback que pueden integrar en sus redacciones finales (E1, E2, E3).

El grueso de la asignatura queda integrado por el diseño y desarrollo del proyecto de trabajo, que se convierte en un proyecto en sí mismo para el alumnado universitario, de tal forma que se realizan aprendizajes globales de todas las áreas de conocimiento: ciencias experimentales y sociales; lengua; matemáticas; psicología; diseño curricular; entre otras. Al ser un proyecto de trabajo colaborativo aprenden a autorregularse, tomar decisiones, organizar el trabajo, etc. La realización de rol-playing les invita a mejorar sus habilidades musicales vocales e instrumentales. 
La puesta en práctica de una actividad en una sesión con niños les ayuda a contextualizar sus prácticas en la realidad contextual y a realizar adaptaciones. Todas las propuestas son guiadas siguiendo los principios de los Pedagogos Creativos, de tal manera de las actividades fomentan en el alumnado su capacidad creativa más que reproductiva, pues los estudiantes componen, arreglan, instrumentan e improvisan con voz, cuerpo e instrumentos y cuerpos sonoros.

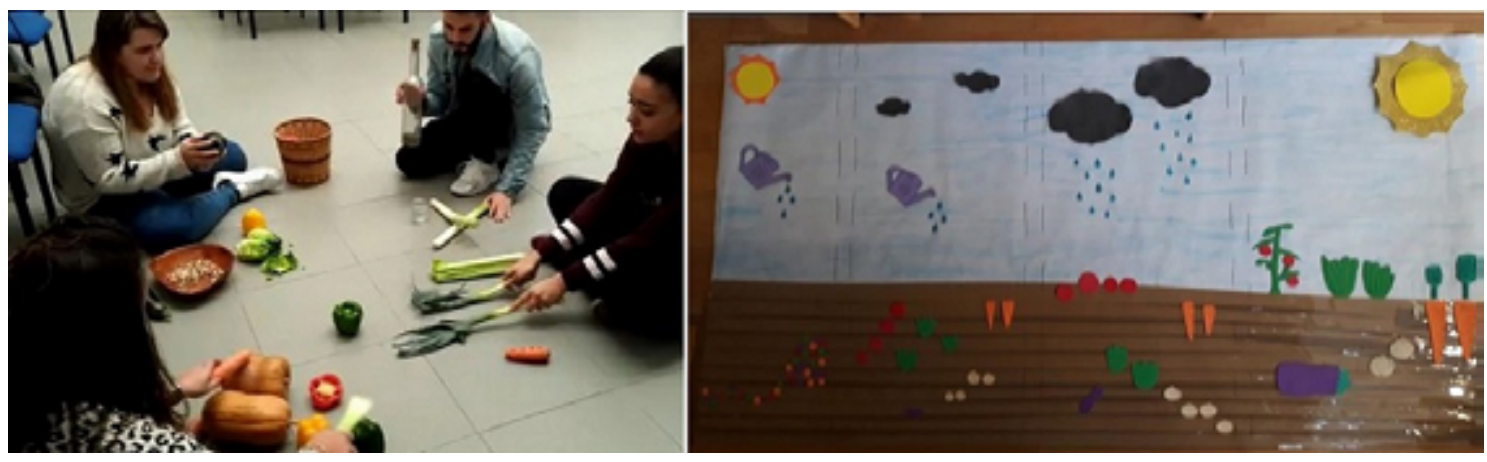

Figura 1. Proyecto Huerto: orquesta vegetal inspirada en la idea de cuerpos sonoros y partituras no convencionales. Fuente: Estudiantes de $4^{\circ}$ curso de Educación Infantil.

\subsection{Proyectos para maestros de primaria}

Se propone la creación de proyectos audiovisuales como ejercicio final de la asignatura "Creación Artística, Cultura Visual y Musical", asignatura compartida con el área de Expresión Plástica. Como pautas generales se plantean las siguientes: formar equipos compuestos por 4-5 integrantes, no superar los 5 minutos en la duración de cortometraje, se podrá utilizar cualquier tipo de técnica audiovisual (cortometraje de ficción, documental, stop-motion, anuncio publicitario, informativo, performance, entre otros) y, por último, se admitirá el uso de cualquier programa de vídeo, cámaras y software para la edición. Respecto al contenido, se propone la necesidad de reflejar un tema actual, "narrado" desde un punto de vista crítico y siguiendo la perspectiva audiovisual. En cuanto a la banda sonora musical se sugiere crear una música original o recurrir a material preexistente, siempre en sinergia con la narrativa del cortometraje.

Las fases propuestas son las siguientes:

- Fase 1. La elección del tema. Una vez conformados los equipos de trabajo, la primera tarea consiste en seleccionar un tema pertinente, de actualidad, acorde a los intereses del grupo y que refleje un punto de vista propio y razonado sobre situaciones dadas en su contexto. Los grupos se reúnen en una o dos sesiones para proponer y debatir ideas, una especie de brainstorming, hasta que definen de manera consensuada el tema central del cortometraje (M3). Esta elección aparece, unas veces, influida por contenidos abordados en la asignatura, por ejemplo, la omnipresencia del lenguaje audiovisual en la actualidad, la importancia de "leer" y asumir de manera crítica los códigos mediáticos, expandir las referencias culturales o valorar el impacto de esta problemática en la educación (M4). Otras veces, las temáticas surgen de las preocupaciones del alumnado sobre su entorno 
y sugiere una postura personal sobre cuestiones educativas, de políticas de género, interacción social, adicciones o comportamientos inherentes a su realidad inmediata.

- Fase 2. Planificación del trabajo. Los equipos asumen diferentes formas organizativas, al menos dos son las más recurrentes: trabajar juntos y de modo simultáneo en las mismas tareas o, de otro lado, distribuir las sesiones de trabajo y designar un responsable para cada una de ellas. En ambas modalidades se asignan roles a cada integrante del grupo. Las etapas fundamentales consisten en: establecer el guión -en algunos casos, acompañado por un storyboard, que permite precisar las diferentes escenas y tiempos-; la grabación; la edición y el visionado del material audiovisual (M1).

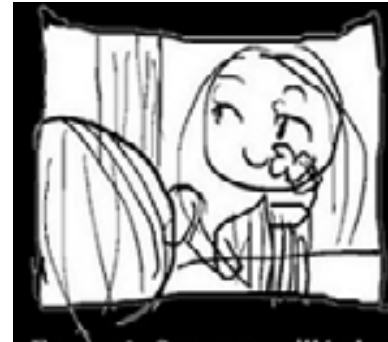

Escena 1: Saray maquillándose Plano: Primer plano

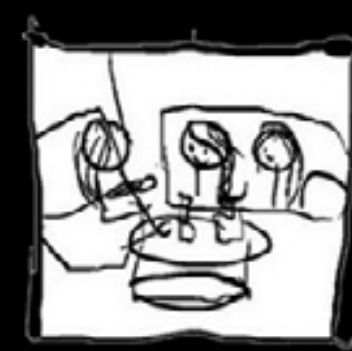

Escena 4: Hablando y llaman

al timbre

Plano: General

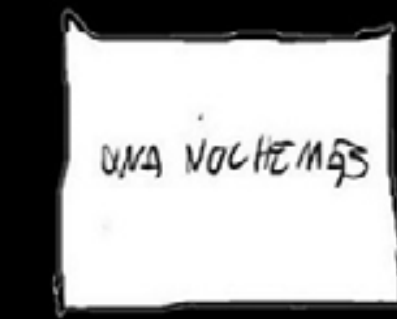

Escena 2: Titulo

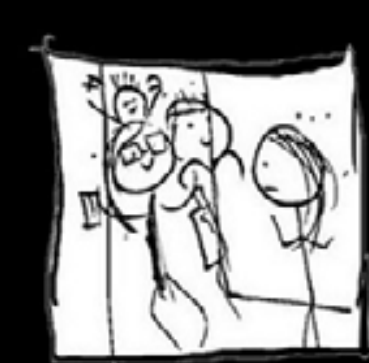

Escena 5: Llegan Jorge, Clara y

Plano: Americano

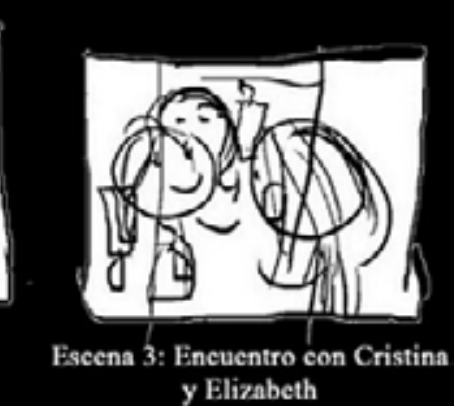
y Elizabeth

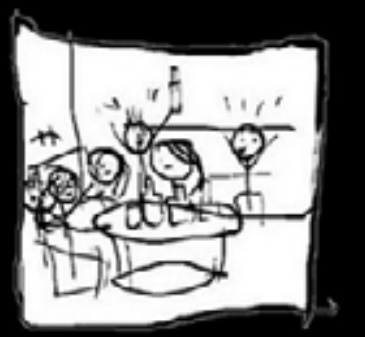

Escena 6: Comienza la fiesta Plano: General

Figura 2. Fragmento del storyboard de "Una noche más" (2017).

Fuente: Proyecto realizado por estudiantes de $3^{\circ}$ curso de Educación Primaria.

- Fase 3. Desarrollo. Una vez definido el guion con las ideas esenciales del cortometraje, se trazan las escenas y se graban. En esta fase se diversifican los roles: unos sirven como actores, otros filman, alguno sostiene la luz y, en algunos casos, implican a compañeros de otros grupos para que actúan como extras o intervengan en tareas técnicas. La etapa más compleja del proyecto es, sin duda, el proceso de edición. Algunos estudiantes cuentan con mayor competencia tecnológica y aportan su experiencia al resto del equipo. La edición compromete el resultado final del proyecto e implica conocimientos sobre el montaje y tratamiento de imágenes y sonido, la sincronización, el uso de efectos digitales, entre otros. Los grupos se reúnen para el visionado del cortometraje, transforman alguna escena, reducen o amplían cierto diálogo, agregan efectos, revisan los créditos finales, hasta que el material queda listo (M5). 
- Fase 4. Evaluación. Los equipos entregan el material audiovisual en soporte digital (CDDVD), acompañado de un texto breve que refleja, sucintamente, los siguientes datos: título del cortometraje, breve justificación de la temática elegida, músicas utilizadas (título, autor, fuente) y, en el caso de imágenes no originales, la referencia correspondiente (M2). La evaluación es compartida y consensuada entre el profesorado -música y plásticaque imparte la asignatura. Se toman en consideración la originalidad y el tratamiento adecuado de los temas, el resultado estético del audiovisual, el correcto uso de la banda sonora, el trabajo colaborativo, entre otros aspectos (E3).

Este tipo de creaciones evidencia el posicionamiento de los jóvenes respecto a los temas que seleccionan y el sentido crítico de sus propuestas. De los últimos tres cursos suman 69 cortos educativos, entre los cuales prevalecen líneas temáticas como: el uso y abuso de la tecnología digital (adicciones tecnológicas, cyberbullying); problemáticas del sistema educativo (crítica a los modelos tradicionales, exceso de deberes escolares, vínculos entre familia y escuela); cuestiones de género (violencia de género, diversidad sexual); ecología y respeto a la diversidad cultural. Estos proyectos favorecen la búsqueda de estrategias creativas en el alumnado, el desarrollo de competencias tecnológicas, de trabajo cooperativo, la interdisciplinariedad y el sentido crítico como ejes fundamentales para la formación de los futuros docentes.

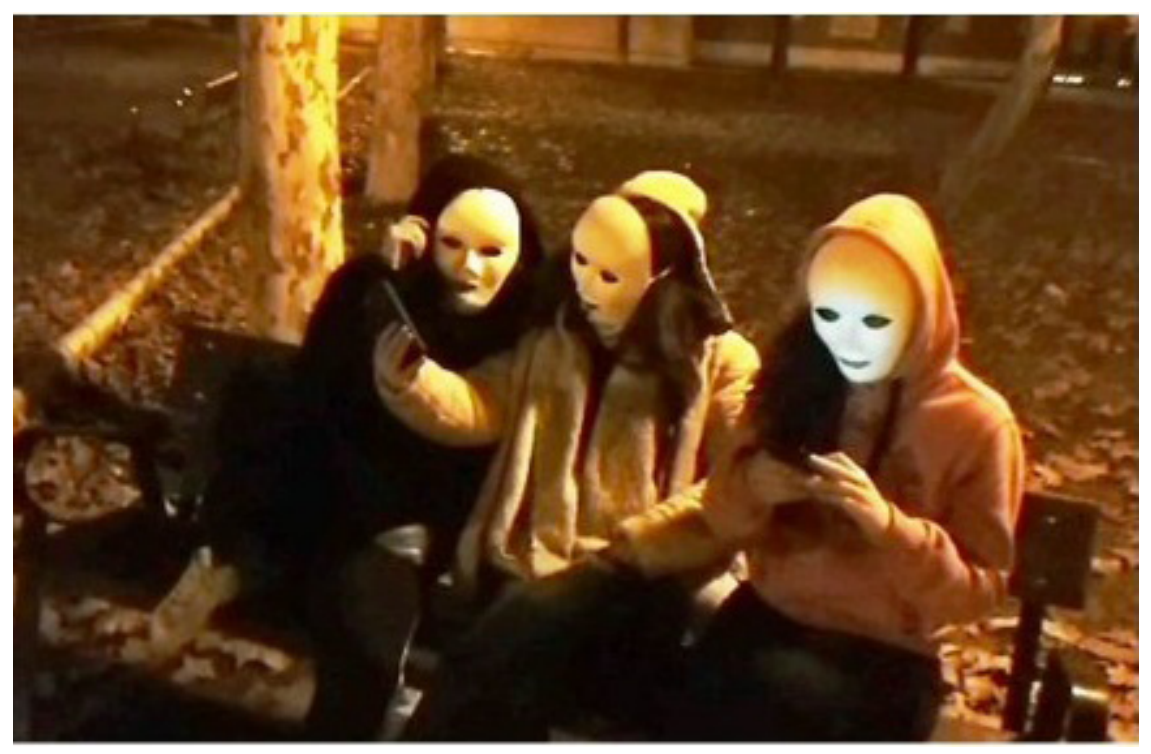

Figura 3. Imagen del cortometraje "Desconexión" (2018). Fuente: Proyecto realizado por estudiantes de $3^{\circ}$ curso de Educación Primaria.

\subsection{Proyectos para la educación social}

El proyecto que se realiza en la asignatura "Técnicas de dinamización Social" consiste en la creación de talleres para un encuentro anual que se realiza en la Facultad de Educación de Palencia llamado “Jornadas Creciendo en Educación”, que en el curso 2019-2020 alcanzará su cuarta edición. 
Durante estas jornadas se invita a niños de la Orquesta In Crescendo, orquesta que surge en el curso 2010-2011 como iniciativa de la Orquesta Sinfónica de Castilla y León, a través del Área Socioeducativa, inspirada en el Sistema de Orquestas Infantiles y Juveniles de Venezuela, con la finalidad de fomentar valores de convivencia, cooperación e integración. Dicha orquesta se ubica en el CEIP Antonio Allue Morer, de Valladolid, que por sus características y trayectoria de trabajo se consideró el centro más idóneo.

Este proyecto, que lleva en marcha más de nueve años y es el único proyecto de estas características en un colegio público en España tiene un importante impacto en la comunidad (Valles, 2018). Los niños del colegio, en su mayoría de etnia gitana y migrantes han aprendido música y a interpretar un instrumento musical además de mejorar su rendimiento académico y su participación escolar; las familias de los niños han descubierto un mundo diferente a través del seguimiento de los conciertos de sus hijos; los profesores de la Orquesta Sinfónica de Castilla León han descubierto una nueva forma de hacer música, desde un lado más intuitivo, cercano, democrático y participativo; el propio Centro Educativo, un colegio muy activo que se ha visto enriquecido por la música y por infinidad de proyectos de colaboración con diversas instituciones, entre ellas la Universidad; y por último, la comunidad también se ha visto enriquecida con conciertos en sus salas y sus calles que incluyen una participación más democrática, lúdica y participativa. El alumnado diseña, planifica e implementa talleres para los niños en ocho talleres paralelos rotativos. Después se celebra un concierto en el que participan niños de los colegios Allue Morer y otros centros educativos de la zona, además de estudiantes y profesorado de la Facultad de Educación.

- Fase 1. La elección del tema. El reto de los estudiantes del grado de Educación Social es doble: por un lado, deben realizar talleres para niños en riesgo de exclusión social, normalmente tras haber pactado las necesidades con profesores y equipo directivo del centro; y por otro lado deben implicar a la comunidad: otras asociaciones, comunidad universitaria, etc. El tema es libre pero siempre que fomente la creatividad y se adapte a esas necesidades (M3).

- Fase 2. Planificación del trabajo. Se aprovechan algunas clases presenciales de la asignatura para diseñar, planificar y perfeccionar los talleres (M4). Desde el punto de vista organizativo, todo gira en torno a la fecha elegida para el encuentro. El alumnado diseña los talleres y a través del role-playing los ensaya con los compañeros para mejorarlos antes de la fecha (M2, M5).

- Fase 3. Desarrollo. La intervención se realiza a dos niveles: la realización de talleres y la celebración de un concierto para implicar a la comunidad (M5).

Los talleres están pensados para estos niños en riesgo de exclusión social, con temáticas distintas. Algunos de los talleres que se han organizado han sido: taller de beatboxing y rap; taller de sombras con temática social; taller de audiovisual; taller de mesa de luz; taller de percusión corporal; taller de instrumentos reciclados, entre otros. Tras los talleres se almuerza todos juntos. Después se hace un concierto en el que participa la comunidad educativa (alumnos, profesores) y niños de otros centros educativos, personas con discapacidad, etc. 
- Fase 4. Evaluación. La evaluación del proyecto se hace a varios niveles: una evaluación informal a través de foros de discusión que diseñan los estudiantes y otra evaluación más formal, pequeñas entrevistas a profesorado del centro y autoevaluación que tome en consideración todos los niveles de acción del proyecto (E3).

La organización de estas jornadas implica todo un desarrollo de competencias profesionales para los estudiantes que incluye: reservar aulas, pedir permisos, solicitar espacios, hacer cuñas de radio, publicar notas de prensa, etc. Por otra parte, deben ponerse en contacto con diversas instituciones: centros educativos, fundaciones y asociaciones. Deben organizar toda la logística del día de la jornada: recepción, traslados de los niños, usos de materiales, almuerzo, organización para el concierto, realización de los talleres propiamente dichos con contenido musical, etc. Por último, deben concluir la jornada, recoger, y evaluar. El contacto con una situación educativa real con niños constituye una importante fuente de motivación para ellos, sobre todo porque se trata de niños en riesgo de exclusión social.
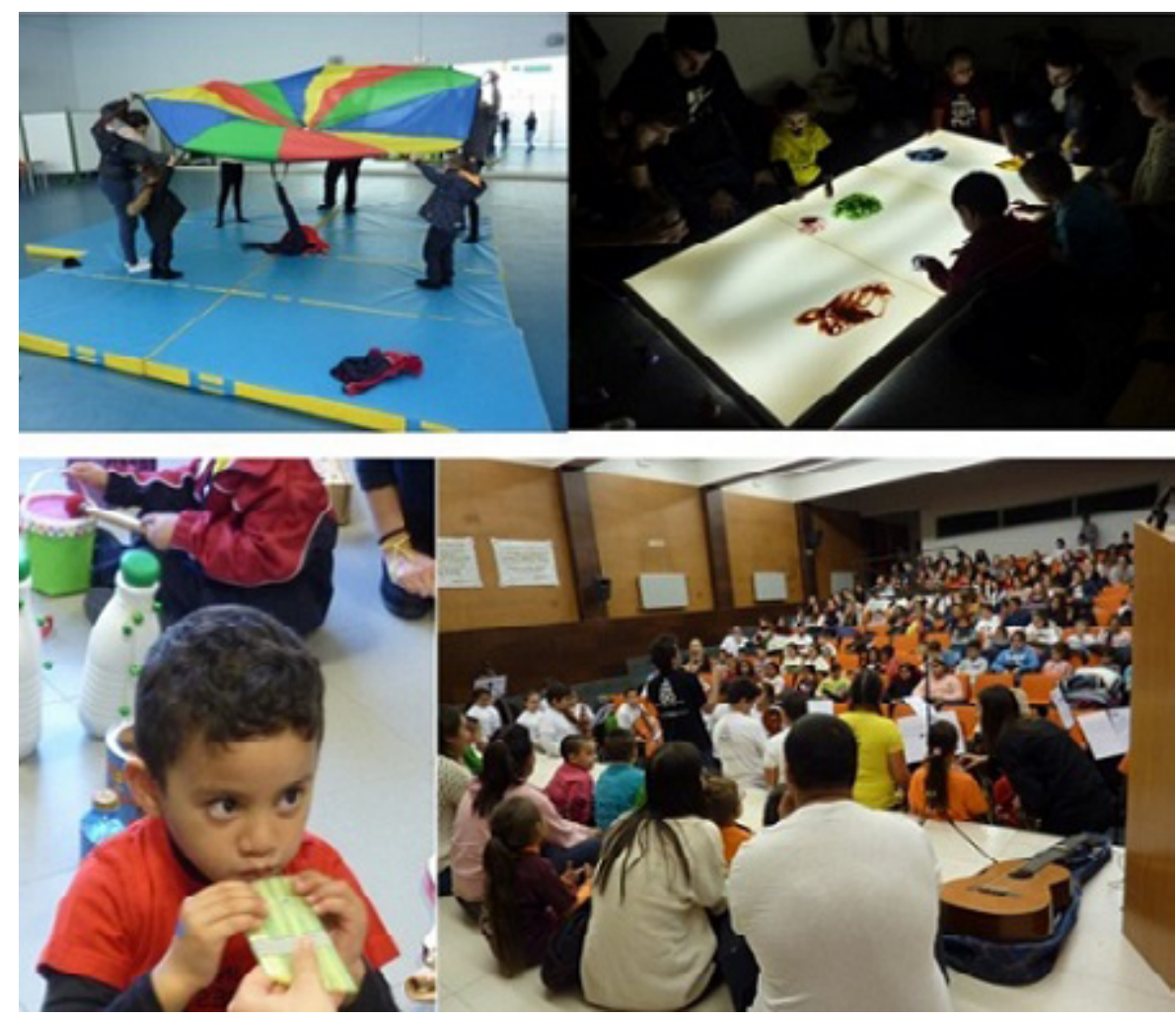

Figura 4. Imágenes de las Jornadas Creciendo en Educación.

\section{Conclusiones}

Son diversos los retos que afronta la educación en el siglo XXI, atravesada por contextos globales y transmediáticos que desafían los modelos educativos y culturales. Sin duda, la educación musical contribuye con estrategias propias a afrontar esta realidad. La formación en música de los futuros 
educadores constituye un eslabón básico en ese propósito, el cual, más allá de proveer únicamente los fundamentos y recursos de las materias musicales, debe además fomentar enfoques renovados y comprometidos con el presente, más próximos a la realidad de las aulas.

En este trabajo se han identificado tres retos que hacen frente a los contextos educativos actuales: el componente creativo de la educación, la responsabilidad social y el uso de las TICs de forma crítica. Las propuestas creativas de Paynter (1999), Schafer (1976; 2013) y Alcázar (2013) entre otros autores, permiten desplegar diversas herramientas compositivas, de performance e improvisación musical que, a su vez, invitan a estrechar vínculos con otros lenguajes artísticos y tecnológicos que conviven en la contemporaneidad. Partiendo de esos presupuestos, se muestra la experiencia pedagógico-musical desarrollada en la Facultad de Educación de Palencia, a través de proyectos realizados por el alumnado de los Grados de Educación Infantil, Primaria y Social. Dicha metodología se fundamenta en el trabajo por proyectos, que convierte a los estudiantes en protagonistas de su aprendizaje y permite desarrollar ideas que fomentan la creatividad, el compromiso social y el uso consciente de las tecnologías tomando como escenario la clase de música.

Los resultados de las autoevaluaciones del alumnado, de las evaluaciones de los profesores a través de todos los medios mencionados permiten corroborar que este camino es efectivo para el desarrollo de competencias creativas, de trabajo en equipo, toma de decisiones, actuación de forma autónoma, entre otras, y se convierte en una manera eficaz de proponer alternativas nuevas, integradoras y significativas en el ámbito de la educación musical. Asimismo, el alcance social de la educación fundamenta la acción comunitaria y la implicación del alumnado universitario con los diversos colectivos, hecho que amplía el espectro de "lo musical" y provee una mayor conexión con la realidad (Peñalba, 2018) y con el compromiso social ciudadano. Por último, el diseño de proyectos a partir de recursos digitales y tecnológicos amplía las estrategias de selección, mediación y uso crítico de estos medios emplazados dentro de entornos creativos y reflexivos en sintonía con los nuevos lenguajes a los que están habituados nuestros estudiantes en el ámbito de la Educación Superior.

\section{Referencias bibliográficas}

Alcázar, A. (2013). Seis invitaciones para una educación musical creativa. En J. Gustems (Ed.), Creatividad y educación musical: actualizaciones y contextos (pp. 16-31). Barcelona: DINSIC Publicacions Musicals.

Aróstegui, J. L. (2003). On the nature of knowledge: What we want and what we get with measurement in music education. International Journal of Music Education, 40(1), 100-115. https://doi.org/10.1177/025576140304000108

Blanco-García, Y. (2018). La Pedagogía de Creación Musical: aulas y talleres creativos. Tabanque. Revista Pedagógica, (31), 42-58. https://doi.org/10.24197/trp.31.2018.42-58 
Carrillo, C., y Pérez-Moreno, J. (2019). El impacto en educación musical: de lo retrospectivo a lo prospectivo. In El impacto de la educación musical: una mirada retrospectiva (pp. 117-125). Barcelona: Octaedro.

Conejo, P. A. (2012). El valor formativo de la Música para la educación en valores. Dedica. Revista de Educação e Humanidades, 2, 263-278.

Cremades-Andreu, R. (2019). Impacto de la educación musical en jóvenes españoles después de finalizar su etapa de educación obligatoria. En J. Pérez-Moreno y C. Carrillo (Eds.), El impacto de la educación musical: una mirada retrospectiva (pp. 29-48). Barcelona: Octaedro.

Delalande, F. (2004). Musical education in the new technologies age. Comunicar, 12(23), 17-23. https://doi.org/10.3916/C23-2004-04

Delalande, F., Vidal, J., y Reibel, G. (2001). La música es un juego de niños. Buenos Aires: Ricordi Americana.

Dennis, B., y Schultis, J. (1991). Proyectos sonoros. Buenos Aires: Ricordi Americana.

García Pedraza, R. (2015). Desafíos a la igualdad en el siglo XXI: dificultades y retos del modelo de escuela comprensiva. Revista Española de Educación Comparada, (26), 135. https://doi. org/10.5944/reec.26.2015.14445

Giráldez, A. (2010). Repensar la educación musical en un mundo digital. En A. Giráldez (Ed.), Música: complementos de formación disciplinar (pp. 73-100). Barcelona: Graó.

Gustems, J. (Ed). (2013). Creatividad y educación musical : actualizaciones y contextos. Barcelona: DINSIC Publicacions Musicals.

Hallam, S. (2010). The power of music: Its impact on the intellectual, social and personal development of children and young people. International Journal of Music Education, 28(3), 269-289. https://doi.org/10.1177/0255761410370658

Ibarra, M. S., y Rodríguez, G. (2010). Los procedimientos de evaluación como elementos de desarrollo de la función orientadora en la Universidad. Revista Española de Orientación y Psicopedagogía, 21(2), 443-461. https://doi.org/10.5944/reop.vol.21.num.2.2010.11558

Kilpatrick, W. (1918). The project method. The Teachers College Record, 19(4), 319-335.

Lauro, C., y Repoll, M. (2011). El Milagro de Candeal [reseña]. Archivos de Ciencias de La Educación, 5, 187-188.

Majno, M. (2012). From the model of El Sistema in Venezuela to current applications: learning and integration through collective music education. Annals of the New York Academy of Sciences, 1252(1), 56-64.

Majó, F. (2001). Por los proyectos interdisciplinarios competenciales. Aula de Infantil, (57), 5-10.

Maldonado-Pérez, M. (2008). Aprendizaje basado en proyectos colaborativos. Una experiencia en educación superior. Laurus, 14(28), 150-180.

Mansfield, J. (2009). El sujeto musical global, el currículum y el cuestionamiento de la tecnología de Heidegger. En D. K. Lines (Ed.), La educación musical para el nuevo milenio: el futuro de la teoría y la práctica de la enseñanza y del aprendizaje de la música (pp. 169-186). Madrid: Morata.

Martín, M. L. (1998). Fundación Psico-Ballet Maite León. Polibea, (46), 23-28. 
Murillo-Ribes, A., Riaño-Galán, M. E., y Berbel-Gómez, N. (2018). Percepción sobre el uso de "Soundcool" como propuesta de intervención en la creación sonora y en el desarrollo de competencias docentes. Un estudio exploratorio en la formación inicial del profesorado. Psychology, Society and Education, 10(1), 127-146. https://doi.org/10.25115/psye.v10i1.1051

Organización para la Cooperación y el desarrollo. (2004). La definición y selección de competencias clave. Resumen ejecutivo. http://deseco.ch/bfs/deseco/en/index/03/02.parsys.78532. downloadList.94248.DownloadFile.tmp/2005.dscexecutivesummary.sp.pdf

Pareja, J. A. (2011). Modelos globalizadores y técnicas didácticas interdisciplinares. En M.

Lorenzo (Coord.), Didáctica Para La Educación Infantil, Primaria y Secundaria (pp. 167-198). Madrid: Universitas.

Paynter, J. (1999). Sonido y estructura. Madrid: Ediciones AKAL.

Peñalba, A. (2017). La defensa de la educación musical desde las neurociencias. Revista Complutense de Investigación en Educación Musical, 14, 109-127. https://doi.org/https://doi.org/10.5209/ reciem. 54814

Peñalba, A. (2018). Claves para una educación musical temprana, creativa e inclusiva. Tabanque: Revista Pedagógica, 31, 29-41. https://doi.org/10.24197/trp.31.2018.29-41

Robertson, S., y Dale, R. (2008). Las tecnologías de la información y la comunicación como medio, objetivo, referencia y consecuencia para el aprendizaje en Europa y la educación del futuro. En J. L. Aróstegui y J. B. Martínez Rodíguez (Eds.), Globalización, posmodernidad y educación : la calidad como coartada neoliberal (pp. 143-166). Madrid: Akal Ediciones.

Savage, J. (2007). Reconstructing Music Education through ICT. Research in Education, 78(1), 65-77. https://doi.org/10.7227/RIE.78.6

Schafer, R. M. (1994). The soundscape : our sonic environment and the tuning of the world. Rochester, Vermont: Destiny Books.

Schafer, R. M. (2013). El paisaje sonoro y la afinación del mundo. Barcelona: Intermedio ediciones.

Serrano, R. M. (2017). Tecnología y educación musical obligatoria en España: referentes para la implementación de buenas prácticas. Revista Electrónica Complutense de Investigación en Educación Musical - RECIEM, 14(0), 153-169. https://doi.org/10.5209/RECIEM.54848

Serrano, R. M., y Peñalba, A. (2019). Las características del profesorado como factores de impacto en la educación musical obligatoria. En J. Pérez-Moreno y C. Carillo (Eds.), El impacto de la educación musical: una mirada retrospectiva (pp. 101-116). Barcelona: Octaedro.

Suárez, M. (2000). Las corrientes pedagógicas contemporáneas y sus implicaciones en las tareas del docente y en el desarrollo curricular. Acción Pedagógica, 9(1), 42-51.

Valles, M. J. (2018). La orquesta entra en la escuela. "In crescendo": Luces y sombras de un encuentro prometedor. Tabanque: Revista Pedagógica, 31, 108-122. https://doi.org/10.24197/ trp.31.2018.108-122

Ventura, M. (1996). Cuadernos de pedagogía. Cuadernos de Pedagogía, (253), 66-71.

Yúdice, G. (2017). La diversidad musical en la nube. En L. Albornoz y M. T. García (Eds.), El audiovisual en la era digital : políticas y estrategias para la diversidad (pp. 105-135). Madrid: Cátedra. 